\title{
LA AGROECOLOGÍA: UN MARCO DE REFERENCIA PARA ENTENDER SUS PROCESOS EN LA INVESTIGACIÓN Y LA PRAXIS
}

\author{
JHON JAIRO MONJE CARVAJAL ${ }^{1}$ \\ jhonmonje@gmail.com
}

Manizales, 2010-04-25 (Rev. 2011-01-17)

\section{RESUMEN}

La Agroecología es un referente teórico, que sirve de orientación general para las experiencias de agriculturas ecológicas, desde el ámbito local, para el fortalecimiento de los sistemas de producción, con un respeto por las estructuras ecológicas y sociales. Desde este fundamento, se generó la necesidad de ganar espacios en la academia y las instituciones de investigación, para enriquecer las bases epistemológicas de la Agroecología a través de la investigación científica, buscando hacer un cambio del paradigma científico. Se plantea la Agroecología como un movimiento social, sin desconocer que los resultados cimientan los soportes para las investigaciones científicas de la academia. Entonces, la interacción entre la investigación en Agroecología y los movimientos agroecológicos son un desafío para ésta, que hasta ahora no ha planteado estudios sin que haga una división entre el modelo reduccionista del paradigma científico convencional, y los enfoques sistémicos y holísticos de la Agroecología, buscando considerar estudios integrados que circulen en la complejidad de los sistemas naturales y sociales. Para entender los enfoques de la Agroecología y el planteamiento de la investigación, se hace necesario conocer las bases conceptuales. Así la Agroecología como planteamiento investigativo tiene la premisa de tener una formulación social, que se sustente en movimientos participativos para la construcción de conocimientos a partir de experiencias ancestrales o principios de coevolución social y ecológica.

\section{PALABRAS CLAVE:}

Agroecología, agricultura alternativa, desarrollo rural, ciencia post-normal, investigación campesina.

\section{AGROECOLOGY: A FRAMEWORK OF REFERENCE TO UNDERSTAND ITS INVESTIGATION AND PRACTICE PROCESSES}

\section{ABSTRACT}

Agroecology is a theoretical referent that serves as general orientation for the ecological agricultural experiences from the local sphere for the strengthening of production systems with all due respect for the ecological and social structures. From this foundation, the need to gain spaces in academic fields and research institutions has generated, in order to enrich the epistemological bases of Agroecology by means of scientific investigation, searching for a change in the scientific paradigm. Agroecology is considered a social movement without ignoring that the results consolidate the support for academic scientific research. Consequently, the interaction between research in Agroecology and the agro-ecological movements are its challenge that, so far, has not expressed the need for studies without making a division between the reductionist methods of the conventional scientific paradigm and the systemic and holistic approaches to Agroecology, searching for consideration of integrated studies circulating in the complexity of natural and social systems. In order to understand the Agroecology approaches and the research formulation, it is necessary to know the conceptual bases. Thus, Agroecology as a research approach has as a premise to have a social formulation supported in participation movements for the construction of knowledge from ancestral experiences or social and ecological co-evolution principles.

KEY WORDS: Agroecology, alternative agriculture, rural development, post-normal science, farmer investigation.

\section{AGROECOLOGÍA Y EVOLUCIÓN HUMANA, LA BASE PARA RETOMAR EL CAMINO}

En las teorías de la evolución humana, la agricultura marca un punto importante en el desarrollo de su progresivo acercamiento al ascenso de su forma y modelo de vida, y éste es resultado de la repetición constante de las pruebas de ensayo del aprendizaje en el acierto y el error, que determinó un alto grado de amaestramiento y de integración del hombre con los recursos naturales de su entorno. De esta forma la domesticación, manejo y dominio de semillas se da no por la necesidad de alimentarse el hombre, se da por la necesidad de alimentar sus animales domesticados, es decir, 
la ganadería es más antigua que la propia agricultura, pero le permitió posteriormente ocuparse de la producción de su propio alimento en un lugar y periodo determinado (ver Gráfico 1).

Gráfico 1. La evolución del conocimiento en la prueba de ensayo en acierto o error.

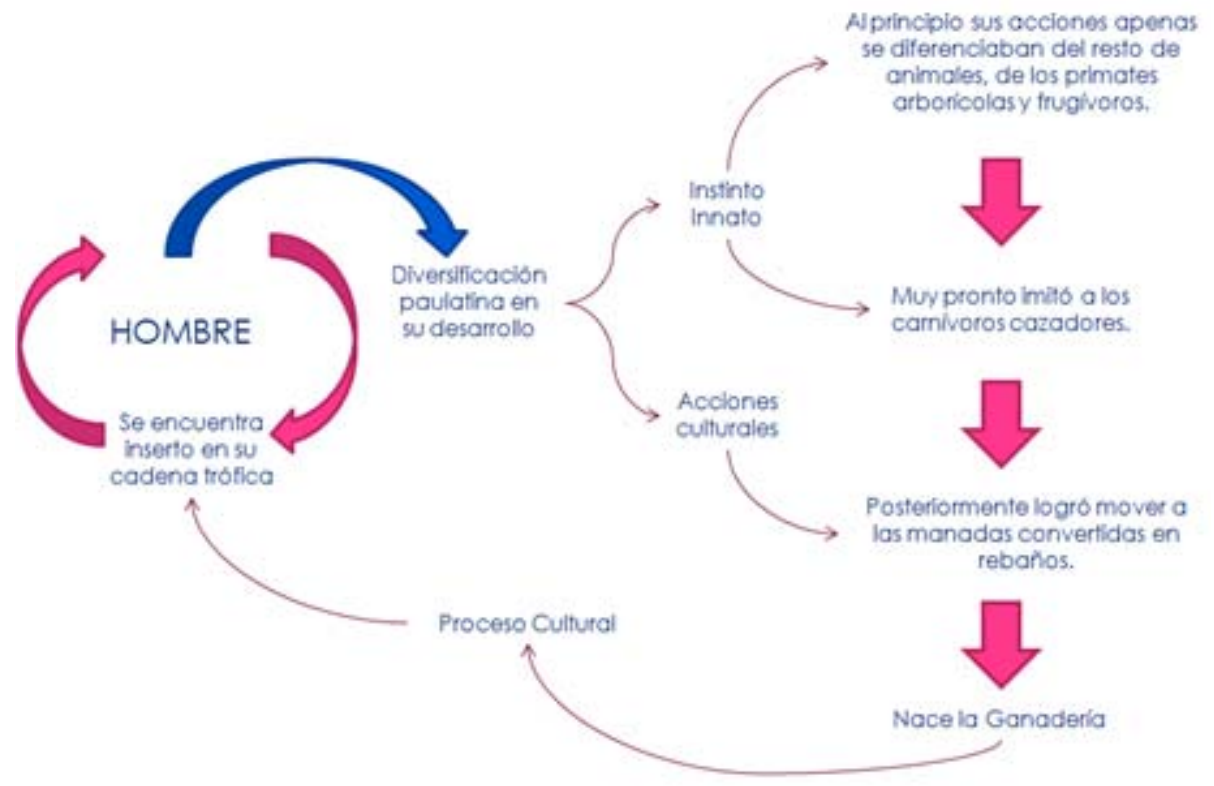

Fuente: Diseño propio (basado en Montserrat, 1988).

Muchos criterios determinan esta verdad, como el hecho de que los productos vegetales eran de fácil recolección, y el grado adaptabilidad del hombre se daba de acuerdo a su movilidad, indicador que no se aplicaba igual a los animales; los cuales eran fundamentales para el ser humano por el arrastre de carga, el suministro de proteína, pieles y en mucho de los casos leche. Estos animales generalmente manejaban una dieta muy localizada en determinadas regiones o zonas, que diferían en cada desplazamiento del hombre, sumándole las dificultades del clima y las respectivas complicaciones de la caza. Estrategias que tempranamente serían sustituidas por la domesticación de los mencionados animales y el aprendizaje del manejo de las semillas.

Es obvio, entonces, que la evolución humana es como una historia de las técnicas agrarias y agropecuarias, directamente ligadas y paralelas en su desarrollo. Parece factible que los sistemas con animales serían los iniciales, pero que muy pronto coexistieron con otras culturas sedentarias, cerealistas; la evolución del cazador por una parte y la del recolector de frutos por otra, en el contexto bíblico por ejemplo a Caín y Abel, como de manera puntual se muestra en el libro sagrado (Montserrat, 1988).

La universalidad del conocimiento que se origina a partir de la relación hombre-naturaleza en la agricultura y la ganadería, data de una archivo histórico de aproximadamente 10.000 años, registrado en la memoria auténtica de campesinos, indígenas y comunidades nativas, que establece un gran número de simbologías, procesos, acciones, herramientas, aprovechamientos, insumos propios y manejos que les permitieron coexistir y coevolucionar en un constante aprendizaje de sus formatos, en el papel ecológico de cada componente.

En este transcurso, el ser humano en los últimos 70 años ha logrado un "dominio" aparente de la naturaleza, dominio que se ha contextualizado y construido a partir de teorías económicas, generando una seudoagricultura; que determinan premisas de estandarización de los sistemas de producción, de homogenización de los cultivos, de utilización de altas demandas energéticas externas en la adecuación de tierras, para hacer suficiente los requerimientos nutricionales de los sistemas agrícolas de producción. Pero en este mismo proceso se ha desconocido de una forma parcializada los antecesores históricos del aprendizaje de más de 10.000 años en la agricultura y ganadería, agregado a este movimiento "verde" un absoluto desconocimiento de los principios ecológicos que rigen el funcionamiento de los ecosistemas, derivando en el uso cada vez mayor de pesticidas y abonos sintéticos (Acevedo, 2004), que con su rutina de aplicación genera un creciente deterioro de la fertilidad de los suelos agrícolas, una erosión en el manejo de semilla y una pérdida de la práctica del conocimiento tradicional. 
Como respuesta a los deterioros de la instalación de la seudoagricultura de los modelos económicos, se inicia un cambio en la forma de ver nuestra relación con la naturaleza, los complejos sistemas sociales y los límites que debía tener el desarrollo. Entonces, se da la retoma de los conocimientos históricos campesinos, indígenas y comunitarios, que se sostuvieron en muchos de los casos por: la conservación de la cosmovisión de los grupos indígenas como guía de sus tecnologías; o por los agricultores modernos que forzados por el mercado bajaron sus costos de producción, reduciendo insumos externos, y adoptando con ello comportamientos productivos propios del campesinado histórico; o aquellos campesinos que no perdieron su modelo al realizar un manejo en gran medida ecológico, manteniendo sus tecnologías tradicionales aisladas de la dependencia del mercado, del consumo y producción de la "moderna sociedad mayor" (Guzmán, González \& Sevilla, 2000).

El cambio inicia en la sustitución de las estructuras simples de la mal interpretada modernidad, hacia un entendimiento de las múltiples interacciones de los procesos naturales de la vida. De igual forma, se buscaba hacer un análisis del mundo no como un conjunto de objetos aislados, sino como una realidad de interacciones de seres de vida y generadores de vida, y no de seres vivos e inertes solamente, comprendiendo el mundo en términos de sistemas dinámicos y complejos, con interacciones entre los componentes de los sistemas y su entorno.

La Agroecología surge como una ciencia ${ }^{2}$ multidisciplinar para enfrentar los problemas causados por la seudoagricultura, y los conceptos de desarrollo económico aplicados a estos sistemas de producción, iniciando una construcción de los referentes conceptuales, integrados en definiciones desde la sociología, la antropología, la agronomía, la ecología y la política.

En la construcción del concepto de Agroecología en su alcance más simple, se sustenta inicialmente que es la aplicación de principios ecológicos al entendimiento y desarrollo de los sistemas productivos sustentables (Altieri y Nichols, 2000; Gliessman, 1998). A partir de los años 90, la Agroecología comienza a integrar, mucho más, conceptos sociales, económicos y políticos en su análisis (Méndez y Gliessman, 2002). La Agroecología, entonces, puede ser definida como el manejo ecológico de los recursos naturales a través de formas de acción social colectiva que presentan alternativas a la actual crisis de modernidad, mediante propuestas de desarrollo participativo, desde los ámbitos de la producción y la circulación alternativa de sus productos, pretendiendo establecer formas de producción y consumo que contribuyan a encarar la crisis ecológica y social, y con ello a restaurar el curso alterado de la coevolución social y ecológica definición construida por Eduardo Sevilla Guzmán (2007).

La Agroecología no nace en ningún grupo científico, ni en ninguna discusión enfocada a la solución de los efectos antrópicos en la naturaleza. Nace en la práctica cultural de los sistemas campesinos históricos en el territorio latinoamericano, nace en el rescate de los conocimientos tradicionales de campesinos e indígenas en América y gran parte de Asia, y como respuesta a los sistemas de producción ecológicas que se daban como una moda en Europa, pero llegando más allá de la concepción meramente productiva.

La evolución de la Agroecología, como una ciencia post-normal (Funtowicz y Ravetz, 2000), la convierte en una herramienta ideal para identificar las bases ecológicas y ambientales de un desarrollo socioeconómico sustentable, haciendo fundamental el incrementar los esfuerzos en el desarrollo de enfoques que realmente integren las realidades sociales, económicas y ecológicas de las zonas donde se implementen sus enfoques. La Agroecología es mucho más que agricultura ecológica y orgánica. Ésta se presenta como una alternativa a los procesos de las seudoagriculturas de los modelos económicos, se enmarca en un movimiento de renovación, que inicia por defender sus bases, que nacen en las agriculturas indígenas, campesinas familiares o comunitarias, y que genera una distancia burocrática lógica, con el planteamiento político del concepto de desarrollo rural y los espacios ganados por la política convencional en la actualidad.

No es raro que se pretenda confundir la Agroecología con un modelo de agricultura de sustitución, con una adopción de determinadas prácticas o tecnologías agrícolas que solo buscan una oferta de productos "limpios" o "ecológicos". Empero, este tipo de agriculturas ecológicas no siempre aplican plenamente los principios de la Agroecología, ya que parte de estas son orientadas a los nichos de mercados, dejando en un segundo plano las dimensiones ecológicas y sociales (Caporal y Costabeber, 2004). Esto queda claro, y es muy puntual cuando se analiza el desarrollo de las agriculturas ecológicas de "mercado", donde se observan acciones como: simplificación de manejos de cultivos, baja diversidad de elementos de sistemas productivos, baja integración en estos elementos, una determinada especialidad de producción sobre pocos productos, una estructura simple de sustitución de insumos químicos por biológicos y orgánicos, sin ninguna consideración por la inclusión social y la creación de alternativas de renta para los agricultores más pobres (Canuto, 1998). En otros términos, una agricultura más limpia que ecológica para el modelo de mercado actual, prevaleciendo la estandarización de productos por la demanda, y no la prioridad de un mercado más justo con lo social y lo natural. 
Entonces, la Agroecología es un referente teórico, que sirve de orientación general para las experiencias de agriculturas ecológicas, desde el ámbito local, para el fortalecimiento de los sistemas de producción, con un respeto por las estructuras ecológicas y sociales. Desde este fundamento, se generó la necesidad de ganar espacios en la academia y las instituciones de investigación, para enriquecer las bases epistemológicas de la Agroecología a través de la investigación científica, buscando hacer un cambio del paradigma científico. Éste ha sido el caso de las naciones y los pueblos latinoamericanos, que poco a poco han ido buscando la apertura de espacios para estas premisas.

Un espacio ganado en este proceso, es la inclusión de los conceptos y principios en las dinámicas sociales rurales de la Agroecología, con una afectiva aceptación en la academia latinoamericana, que desde ahí aporta a la conformación de una base científica de soporte de la misma. Una clara referencia de estos espacios ganados, más por afecto que por la aceptación de un nuevo paradigma, es la apertura de cursos de formación en diferentes universidades, cursos que van desde las formaciones profesionales a nivel de licenciaturas, ingenierías, hasta cursos de maestría y doctorado (ver Gráfico 2).

Gráfico 2. Distribución geográfica de los diferentes programas de Agroecología en Latinoamérica.

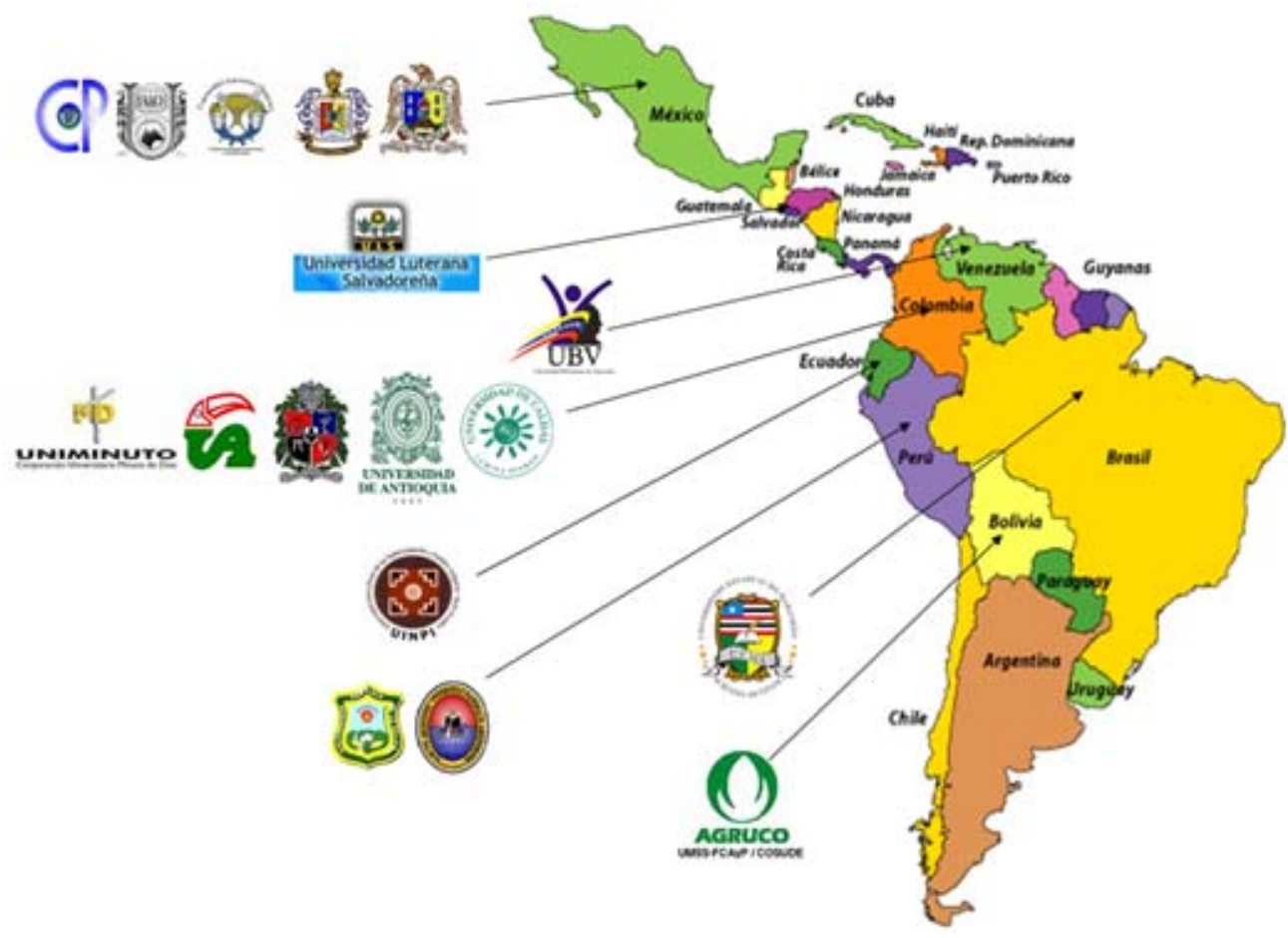

Fuente: Consulta, diseño y construcción propia.

Los beneficios de la aplicación de los principios de la Agroecología, se han visto reflejados en estupendos trabajos, con la replicación de experiencias exitosas en comunidades, generalmente en grupos sociales históricamente marginados. Su base es el bosquejo de un aprovechamiento de recursos locales, de coexistencia y coevolución ecológica y social. En este caso se plantea la Agroecología como un movimiento social, sin desconocer que los resultados cimientan los soportes para las investigaciones científicas de la academia. Se plantea, entonces, que la interacción entre la investigación en Agroecología y los movimientos agroecológicos son un desafío para ésta, y que hasta ahora no ha planteado estudios sin que haga una división entre el modelo reduccionista del paradigma científico convencional, y los enfoques sistémicos y holísticos de la Agroecología, buscando considerar estudios integrados que circulen en la complejidad de los sistemas naturales y sociales.

El abordaje de este desafío implica para la academia y las instituciones de investigación, un replanteamiento de los métodos, enfoques de los sistemas y modelos de investigación, entendiendo claramente que el paradigma científico de reducción y especialización posee una asombrosa resistencia a las innovaciones, operando simultáneamente en las políticas de investigación de las instituciones y la academia, desde y para sus investigadores. No se podrá, entonces, hacer una 
renovación de los conceptos y enfoques de investigación, sin antes hacer una renovación de los enfoques de los investigadores, y viceversa.

Enfrentar este círculo vicioso, para establecer un proceso de renovación epistemológico y metodológico, no es una tarea trivial en instituciones con una experiencia investigadora fuerte e importante, es más una virtud del proceso de elaboración de una posición institucional oficializando el abordaje de un nuevo marco de referencia, sin que éste sea un planteamiento meramente administrativo; o como una línea de proceder obligatoria, éste debe ser más un proceso de reflexión colectiva, sintonizado con una dinámica emergente y cada vez más aceptada en los componentes de la institución, generando una corriente creciente de investigación en Agroecología, que de forma aislada o en pequeños grupos se va innovando en las prácticas de investigación científica, según los acercamientos del enfoque agroecológico.

El sentido acá planteado, no puede en ningún momento confundir con tentativas o intenciones de descalificar las competencias científicas acumuladas en largos años de trabajo de las instituciones de tradición. Por el contrario, las investigaciones en Agroecología deben estar soportadas en esta tradición científica institucional, con esto se da un sustentáculo científico más fuerte; empero, estas mismas no pueden tener igual formato de investigación del paradigma científico dominante; ésta debe ser renovada, replanteada e innovadora en sus metodologías y resultados.

Para entender los enfoques de la Agroecología y el planteamiento de la investigación, se hace necesario conocer las bases conceptuales. No es el hecho de sentar a los investigadores a pensar sobre qué es la Agroecología y cómo se hace investigación en ella; se estaría cometiendo un error de grandes proporciones, más si es el caso de personas con tradición investigadora en el paradigma científico convencional. El fundamento de esta apertura, es que los más adelantados en este ámbito, puedan presentar un bosquejo inicial de la visión emergente de esta nueva ciencia, y mencionar aspectos importantes del debate sobre Agroecología en el ámbito nacional e internacional. Es también, hacer una discusión primaria en cada institución, para refrendar los puntos de percepción, aceptación y negación de los cuerpos investigadores, identificando los afines, diferentes, ajenos y opuestos, dejando sobre la mesa de trabajo los resultados planteados por los participantes, y que se deben enmarcar sobre los conflictos, los distanciamientos, los aislamientos, la diversidad y la complejidad de estas posiciones (ver gráfico 3).

Gráfico 3. Proceso de formación e investigación en Agroecología.

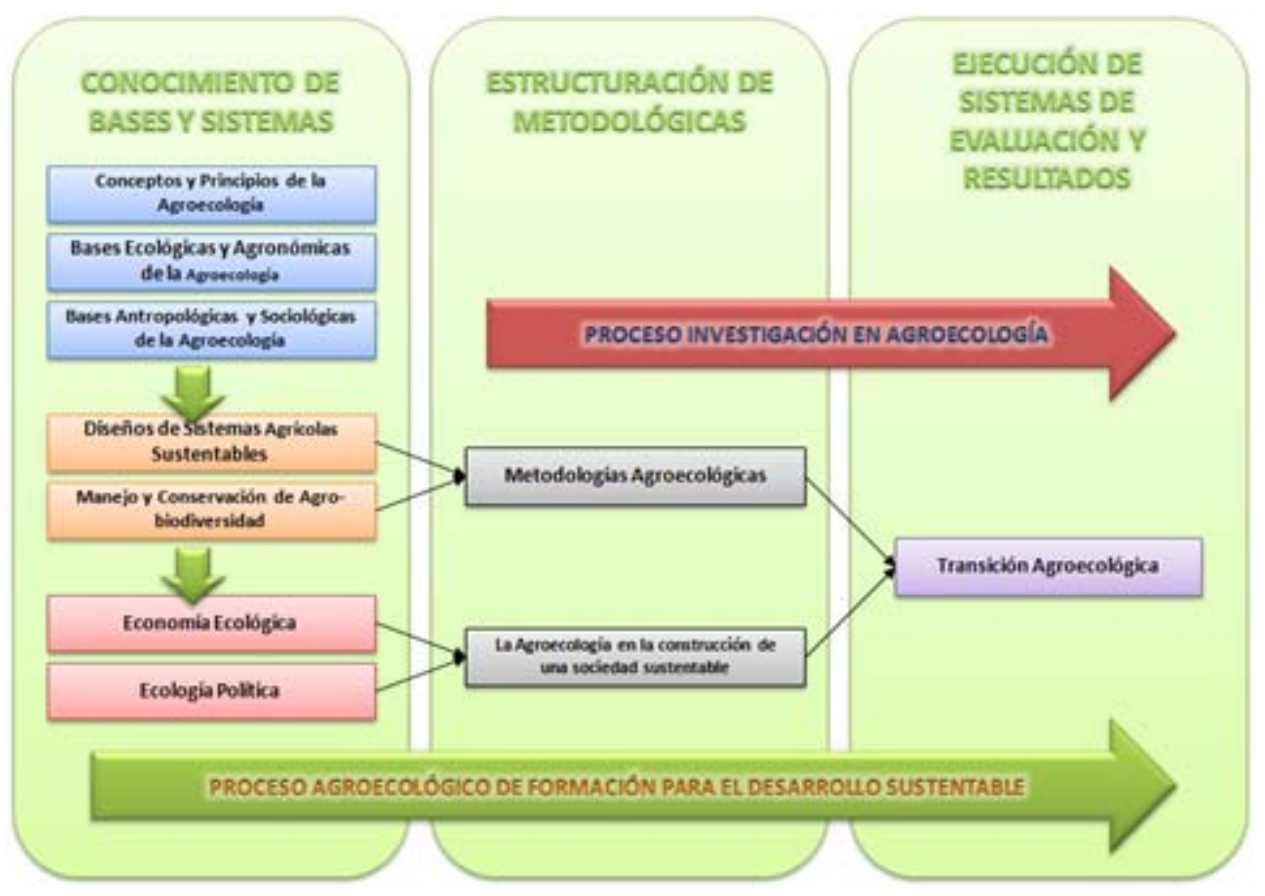

Fuente: La agroecología como ciencia post-normal. Monje (2007).

La Agroecología como tal, no plantea fórmulas o modelos fijos para la investigación; son pautas a las que se les deben agregar planteamientos participativos, reducciones o ajustes, mediante una 
observación sistemática de los modelos productivos, sociales, culturales, que buscan un respeto por las bases de la sustentabilidad. Así la Agroecología como planteamiento investigativo tiene la premisa de tener una formulación social, que se sustenta en movimientos participativos para la construcción de conocimientos a partir de experiencias ancestrales o principios de coevolución social y ecológica. También se propone la generación de conocimientos y métodos innovadores, y estrategias de recontextualización entre los conocimientos ancestrales acumulados a lo largo del tiempo y la generación de nuevos conocimientos.

Pero se puede cometer un error en este proceso al ignorar lo acontecido desde los años 50 con la Revolución Verde y desde los años 70 con la transgénesis, ya que estos momentos han dejado una base de conocimientos, que indican cómo no se deben hacer diseños de sistemas productivos; han dejado una gran muestra en el terreno de los grandes deterioros de los suelos, las aguas y la biodiversidad, y una base conceptual y teórica científica de cómo los modelos comerciales no se ajustan a las realidades naturales, a las dinámicas ecológicas, a las relaciones sociales para lograr una coevolución compartida entre lo socioeconómico y cultural, lo ecológico productivo, y el componente político del ser (Altieri y Nichols, 2000).

Planteado entonces el marco general de las investigaciones desde la Agroecología, haciendo un referente histórico de la evolución de la agricultura y la ganadería, y mencionando los puntos de trabajos que se deben considerar, se deja este esbozo para la academia y los institutos de investigación que buscan o deseen hacer inmersión en esta nueva ciencia, y la posibilidad de que construyan su propia bitácora de navegación, para que luego tomen las rutas recorridas y se desarrolle una línea determinada pero flexible para los planteamientos de la investigación desde y para la Agroecología. Ya al final de cada etapa, se irán conociendo los resultados, pero más que estos, se busca que cada proceso deje un impacto en las comunidades donde se trabaja, que se haga un proceso de transferencia real de conocimiento y que se aporte al desarrollo alternativo para el mejoramiento de su calidad de vida, bajo las dimensiones de la Agroecología y los retos que ésta plantea para las regiones de nuestro territorio (Monje, 2007.

\section{BIBLIOGRAFÍA}

- Altieri, Miguel, y Nichols, Clara. (2000). Agroecología, teoría y práctica para una agricultura sustentable. Programa de las Naciones Unidas para el medio ambiente PNUMA - Red de formación ambiental para América Latina y el Caribe. México D.F.

- Acevedo, Alvaro (2004). Agricultura sutentable. Principios, estrategias y prácticas. Bogotá: Ed La Silueta.

- Canuto, João C. (1998). Agricultura Ecológica en Brasil: perspectivas socioecológicas. Tesis de Doctorado en Agronomía. Universidad de Córdoba. Córdoba. España.

- Caporal, Francisco R., y Costabeber, J. A. (2004). Agroecologia: alguns conceitos e princípios. Brasília D.F., Brasil. Ministério do Desenvolvimiento Agrário - Secretaria da Agricultura Familiar - DATER: IICA.

- Funtowicz, Silvio O, y Ravetz, Jerome R. (2000). La ciencia posnormal: ciencia con la gente. Barcelona, España: Icaria Editorial.

- Gliessman, Stephen. (1998). Agroecología: procesos ecológicos en agricultura sostenible. Boca Ratón, Florida. EE.UU.: Lewis/CRC Press.

- Guzmán Casado, G., González de Molina, M., y Sevilla Guzmán, E. (2000). Introducción a la Agroecología como desarrollo rural sostenible. Madrid, España: Mundi-prensa.

- Mendez, Ernesto, Gliessman, Stephen (2002). Un enfoque interdisciplinario para la investigación en agroecología y desarrollo rural en el trópico latinoamericano. En manejo integrado de plagas y agroecología No 64. Costa Rica. p5-16.

- Monje, Jhon J. (Enero-Junio 2007). Retos de la Agroecología en las regiones colombianas. Revista Luna Azul, 24: 68-73. Manizales: Universidad de Caldas. Obtenido el día 20, agosto, 2010., desde http://lunazul.ucaldas.edu.co/downloads/Revista24_9.pdf

- Montserrat, P. (1988). Los Sistemas Agrarios. Mecanografiado L. Wordpress, 245b.

- Sevilla Guzmán, Eduardo (2006). De la sociología rural a la agroecología. Barcelona España: Icaria Editores.

1. Ingeniero Agroecólogo (Universidad de la Amazonía). Máster en Agroecología, Sociología y Desarrollo Rural Sostenible (Universidad Internacional de Andalucía). Doctorado en Agroecología, Sociología y Desarrollo Rural Sostenible (Universidad de Córdoba, España). Docente Corporación Universitaria Minuto de Dios. Docente catedrático Universidad de los Llanos. 
2. Desde los años 90 se ha estado dando una discusión muy amplia sobre si es una disciplina o una ciencia, sostenida a lo largo de la existencia del programa de Doctorado en Agroecología, en el Instituto de Sociología y Estudios Campesinos en la Universidad de Córdoba en España, que tiene en este momento graduados de la maestría y el doctorado en la mayoría de los países de nuestro continente. El resultado más que demostrar que ésta sea una ciencia o una disciplina, está orientado a que sus enfoques y estudios prevalecen por sobre otros en la mejora y estabilidad de la calidad de vida de los seres de este planeta. 